\title{
Physiopathology of megarectum: the association of megarectum with encopresis
}

\author{
P. MEUNIER, P. MOLLARD, AND J-M. MARECHAL \\ From the Laboratoire d'Exploration Fonctionelle Manométrique et Clinique Chirurgicale Pédiatrique, \\ Hopital Debrousse, Lyon, France
}

SUMMARY Studies of both rectosphincteric reflex threshold and conscious rectal sensitivity threshold were performed on 15 control subjects and 61 children with a radiological megarectum, $70 \%$ of whom were encopretics. In control subjects, the reflex threshold and the sensitivity threshold were obtained with a comparable volume of rectal distension. In the megarectum patients, sensitivity was often considerably reduced, the incidence of encopresis increasing proportionally with the decrease in conscious rectal sensitivity. Patients were segregated in three functional groups, according to measurements of the sensitivity threshold.

During manometric studies of anorectal disorders in children, certain modifications in conscious rectal sensitivity have been noted (Meunier et al., in press). An exaggeration of this sensitivity was observed in Hirschprung's disease. In the normal subject, the anorectal reflexes are accompanied by light conscious rectal sensitivity. A decrease in rectal sensitivity in functional megarectum was often observed by us and by others (Porter, 1961; Callaghan and Nixon, 1964; Lawson, 1975). Because, in children, megarectum is frequently associated with encopresis, the aim of this study was to find a correlation between the frequency of encopresis and a decrease in conscious rectal sensitivity.

\section{Methods}

\section{SUBJECTS}

A total of 76 investigations was performed on 15 control subjects aged 5 to 13 years (mean age $=8.87$ years for seven boys and eight girls), and on 61 children with radiological megarectum, but without aganglionosis, aged 4 to 15 years (mean age $=7.82$ years).

In the latter group, $63 \%$ were boys and $37 \%$ were girls. In the same group, $70 \%$ were encopretics ( $70 \%$ boys and $30 \%$ girls).

Address for reprint requests: Dr P. Meunier, Laboratoire d'Exploration Fonctionelle Manometrique Urinaire et Digestive, Hopita Debrousse, 69322 Lyon Cedex 1 (France).

Received for publication 5 January 1976

\section{TECHNIQUES}

Pressure measurements were performed with a tandem system of three side-opening PVC catheters, placed so that their tips were, respectively, at $10 \mathrm{~cm}$, $5 \mathrm{~cm}$, and $2.5 \mathrm{~cm}$ from the anal margin. Thus, the two upper catheters were in the rectum, and the third was situated in the anal canal. The catheters have an internal diameter of $2.6 \mathrm{~mm}$ and a $2 \mathrm{~mm}$ long side-opening. To avoid occlusion, they were perfused with saline (flow rate $=0.24 \mathrm{ml} . \mathrm{min}^{-1}$ ). Each side-opening catheter was connected to an electromanometer (Hewlett-Packard $1280 \mathrm{C}$ ), the output of which was amplified and registered on a Hewlett-Packard thermal-tip recorder (mod. 7758 A). In addition, a tube (char. 12), on which was mounted a Latex balloon, was attached to our three-catheter probe. The balloon, with little resistance to distension, lay in the rectum 10 to $15 \mathrm{~cm}$ from the anal margin. The three catheters and the tube with the balloon were assembled together with tetrahydrofurane. This probe was rigid enough to be introduced without a proctoscope.

If impacted faeces were present, these were evacuated a few days before the procedure. An enema was often required the evening before the procedure in other subjects. No sedation was administered before the investigation, so that no pharmacological reduction in conscious rectal sensitivity would be produced.

The method described, is suitable only for children old enough to cooperate, for it requires the complete quietness and confidence of the subject 
being tested. The procedure must be performed under the calmest possible circumstances.

The experimental procedure included two steps. Firstly, in order to eliminate the possibility of aganglionosis, the rectoanal inhibitory reflex was studied by distending the rectal balloon with air. The distension volume at which internal sphincter relaxation occurred (reflex threshold) was determined during five trials. Secondly, the distension volume for which an initial transient sensation occurred (conscious rectal sensitivity threshold) was determined in another five trials by questioning the child.

\section{Results}

In control subjects (Table 1), internal sphincter relaxation occurred for reflex threshold of between 5 and $30 \mathrm{ml}$ of distension (mean $=15 \cdot 33$ ).

In children with megarectum (Table 2), the reflex threshold was between 5 and $60 \mathrm{ml}$ of distension (mean $=16 \cdot 64)$. The reflex threshold is, therefore, not modified in this disease with respect to the control.

The conscious rectal sensitivity threshold in normal individuals (Table 1) was found to be between 10 and $30 \mathrm{ml}$ of distension (mean 14.66), while, in children with megarectum, it varied from 10 to $380 \mathrm{ml}$ of distension mean (Table 2). The incidence of encopresis increased proportionally with the decrease in conscious rectal sensitivity (increased threshold).

For the sensitivity threshold measurements, the megarectum patients were segregated into three groups (Table 2). Between 10 and $60 \mathrm{ml}$ of distension $($ mean $=29.00), 32 \%$ were encopretics (group 1); between 70 and $100 \mathrm{ml}$ (mean $=83.85$ ), the incidence

\begin{tabular}{rlll}
\hline $\begin{array}{r}\text { Age } \\
(y r)\end{array}$ & Sex & \multicolumn{2}{l}{ Threshold } \\
\cline { 3 - 4 } \cline { 3 - 3 } \cline { 3 - 3 } & & Reflex & Sensitivity \\
\hline 5 & F & 10 & 10 \\
10 & F & 10 & 10 \\
7 & F & 10 & 10 \\
6 & F & 10 & 10 \\
9 & F & 15 & 10 \\
9 & M & 20 & 10 \\
6 & F & 20 & 10 \\
12 & M & 20 & 10 \\
13 & M & 30 & 10 \\
13 & M & 10 & 20 \\
8 & M & 10 & 20 \\
6 & M & 20 & 20 \\
11 & M & 15 & 20 \\
9 & & 15 & 30 \\
9 & &
\end{tabular}

Table 1 Control subjects

Each value (expressed in millilitres), for reflex and sensitivity threshold, is the average of five trials. of soiling jumped to $82 \%$ (group 2); above $100 \mathrm{ml}$ of distension (mean $=209.33$ ) all children were encopretics (group 3). In group 1, rectal sensitivity, although slightly decreased, should allow normal continence; soiling can thus probably be traced to psychogenic factors. In group 2, rectal sensitivity is greatly decreased. Children in this group who were not encopretics have good sphincteric vigilance associated with good rectal adaptability; they have, therefore, an essentially mechanical constipation. All children in group 3, as well as most children in group 2, were encopretics who presented purely reflex evacuations after the superficial lysis of their faecaloma. This automatic passage of mucus and liquid faeces calls to mind the 'overflow' type of incontinence, because, according to Lane (1975), overflow is the result of distension of the rectum, with reflex relaxation of anal sphincters, as occurs with simple faecal impaction.

\section{Discussion}

Such a study of conscious rectal sensitivity is subjective and therefore debatable, particularly in children. Our results, for megarectum patients, show, nevertheless, a very significant increase in encopresis, which is proportional to the decrease in rectal sensitivity. Reflex activity is entirely normal in these children.

In comparable studies (Porter, 1961; Callaghan and Nixon, 1964), the long-lasting relaxation of both sphincters was studied instead of the rectoanalinhibitory reflex. This seemed more logical, because long-lasting relaxation leads directly to defaecation. We preferred the inhibitory reflex, because its threshold remains very constant, unlike the long-lasting relaxation threshold, which often varies considerably in the same subject, even during the same examination. Furthermore, there were considerable variations in the latter threshold from one individual to the other (see also Ihre, 1974, appendix: Table 2). Similarly, it would have been possible to study the desire to defaecate, like Porter (1961) and Callaghan and Nixon (1964), instead of the initial transient sensation. It should be noted that, during the testing of children, it was very difficult to know, with precision, when the urge to defaecate occurred. The initial transient sensation was much easier to study and the results varied little from one trial to another. The same authors observed a decrease in conscious rectal sensitivity in certain cases of megarectum, but an increase in encopresis with a decrease in rectal sensitivity was not reported in their results. Their work was especially oriented toward the study of defaecatory mechanisms, and, because of the complexity of the physiology of continence and 


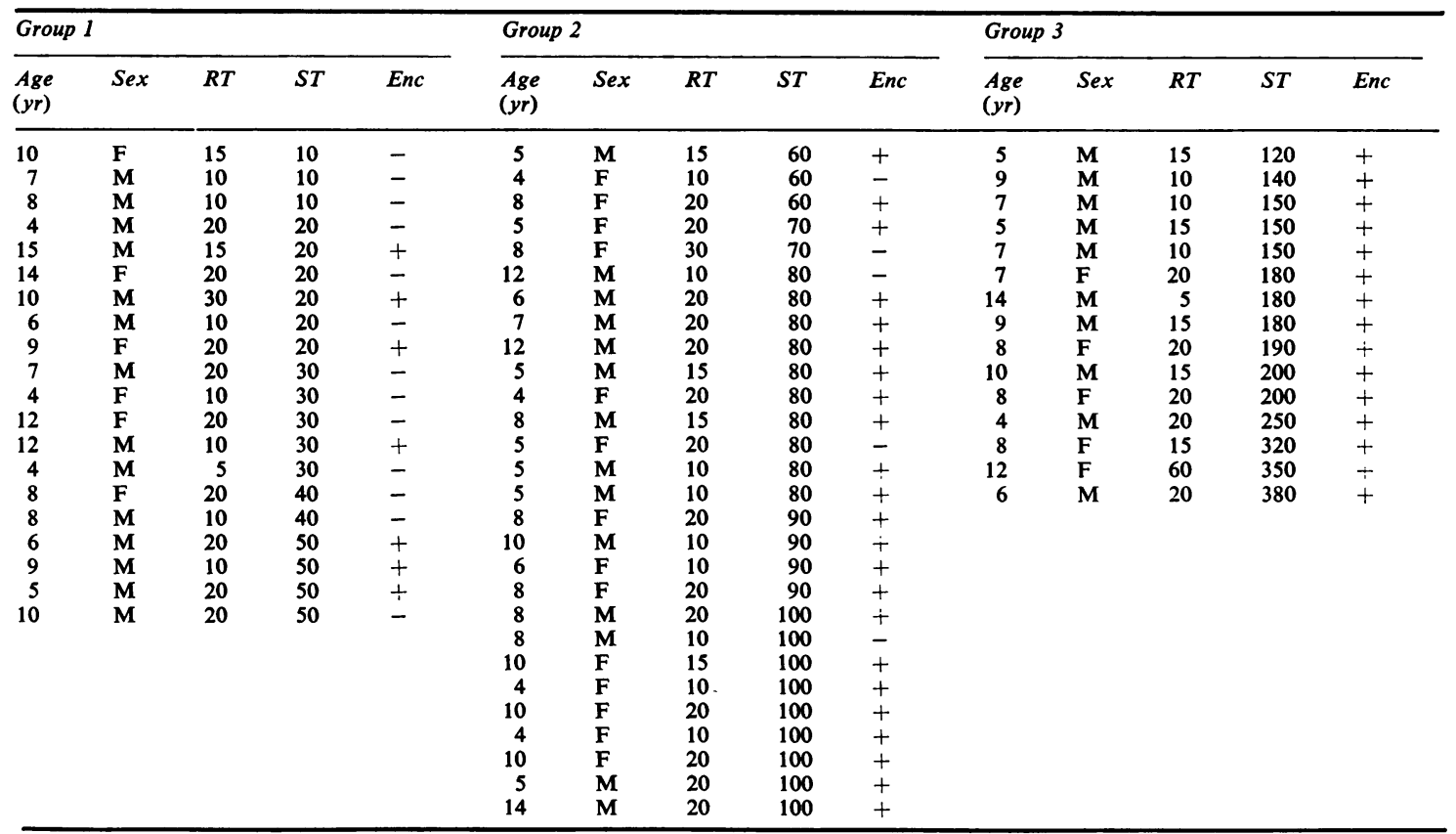

Table 2 Patients with megarectum

Each value (expressed in millilitres) for reflex and sensitivity threshold is the average of five trials. For the refiex threshold, the percentage of variation from one trial to another was less than $20 \%$. For the sensitivity threshold, the percentage of variation was less than $25 \%$.

RT: reflex threshold. ST: sensitivity threshold. Enc.: encopresis. +: yes. $-:$ no.

defaecation (still poorly understood), this approach is certainly less adapted for clinical application.

In the normal individual, the rectoanal inhibitory reflex is controlled by the cerebral cortex, because this reflex has a threshold which is close to that of conscious rectal sensitivity. This control partially disappears in these encopretic children who exhibit purely reflex evacuations. The rectosphincteric apparatus acts automatically in these children, because sacral reflexes function without inhibition. Normal defaecation, which is initiated by conscious rectal sensitivity, is also much more uncommon in these patients.

The natural evolution toward worsening, usual in this disease, is easy to understand. At the onset, for psychological reasons, faecal retention occurs to such degree that impaction results. This faecaloma, distending the rectal wall, lowers the conscious rectal sensitivity and allows soiling. The greater the increase in volume of impacted faeces, the more the rectum is enlarged. Consequently, the sensitivity decreases more and more, and soiling worsens.

Meanwhile, a reflex activity responsible for soiling does not explain why encopresis is much more common in the daytime. However, during sleep, there is an important decrease in rectosigmoid motility (Davidson et al., 1956). Thus, lysis and reflex evacuation of faecal material are lessened.

Although further investigations will be necessary to understand the physiopathology of the motility of the rectal wall in the three groups of megarectum described above, our method is suitable for evaluation of the severity of a megarectum with associated encopresis and for follow-up studies with regard to an established treatment.

We are gratefully indebted to Professor Y. Minaire for his helpful comments and criticism.

\section{References}

Callaghan, R. P., and Nixon, H. H. (1964). Megarectum: physiological observations. Archives of Disease in Childhood, 39, 153-157.

Davidson, M., Sleisenger, M. H., Almy, T. P., and Levine, S. Z. (1956). Studies of distal colonic motility in children. I. Non-propulsive patterns in normal children. Pediatrics, 17, 807-819.

Ihre, T. (1974). Studies on anal function in continent and incontinent patients. Scandinavian Journal of Gastroenterology, 9, suppl. 25.

Lane, R. H. (1975). Clinical application of anorectal physiology. Proceedings of the Royal Society of Medicine, 68, 28-30.

Lawson, J. O. N. (1975). The soiling child. The Practitioner, 
214, 807-808.

Meunier, P., Mollard, P., Jaubert de Beaujeu, M. (1976).

Manometric studies of anorectal disorders in infancy and childhood. An investigation of the physiopathology of continence and defection. British Journal of Surgery (In press).

Porter, N. H. (1961). Megacolon: a physiological study. Proceedings of the Royal Society of Medicine, 54, 1043-1047. 